\title{
Geometrically uniform hyperbolic codes
}

\author{
HENRIQUE LAZARI ${ }^{1}$ and REGINADO PALAZZO JR. ${ }^{2 *}$ \\ ${ }^{1}$ Departamento de Matemática, Universidade Estadual Paulista, UNESP-Rio Claro, SP, Brazil \\ ${ }^{2}$ Departamento de Telemática, FEEC-UNICAMP \\ E-mails: hlazari@rc.unesp.br / palazzo@dt.fee.unicamp.br
}

\begin{abstract}
In this paper we generalize the concept of geometrically uniform codes, formerly employed in Euclidean spaces, to hyperbolic spaces. We also show a characterization of generalized coset codes through the concept of $G$-linear codes.
\end{abstract}

Mathematical subject classification: 14L35, 94B60.

Key words: classical groups, group codes.

\section{Introduction}

The concept of geometrically uniform signal constellations was introduced by Forney in [7]. This concept has been shown to be the most appropriate in the context of Euclidean signal constellations and spaces in the sense of unifying processes such as Ungerboeck set partitioning [14] and generalized concatenation [1].

One of the main objectives of geometrically uniform codes is related to the construction of geometrically uniform partitions and in particular the construction of generalized coset codes.

The objective of this paper is to extend the concepts of signal sets, lattices and set partitioning to the hyperbolic plane by making use of the associated hyperbolic regular tessellations and its corresponding full symmetry groups. In particular, we consider the concept of geometric uniformity for joint modulation and coding processes. Although the hyperbolic isometry groups have greater

*This work has been supported by FAPESP, CNPq, and CAPES, Brazil. 
complexity than the Euclidean isometry groups the procedures and concepts related to the geometrically uniform partitions can be extended to the former. An interesting aspect is that the fundamental regions of the Euclidean regular tessellations always have an Abelian subgroup of order 4 belonging to the corresponding symmetry groups even if the they are not Abelian, whereas in the hyperbolic case more general groups have to be considered. In other words, the existence of regular tessellations in the hyperbolic plane of the type $\{p, q\}$, see Definition 2.1, generating signal sets justify the search for subgroups and quotient of groups associated with the full symmetry group of $\{p, q\}$ which will provide relevant information about the structure of these groups.

The problem that motivated us to consider geometrically uniform codes in the hyperbolic plane is related to the results shown in [5] and [6] with respect to the better performance achieved by a binary communication system using soft-decision decoding (an 8-level quantizer leading to a binary input, 8-ary output symmetric channel, denoted by $\left.C_{2,8}[8,2]\right)$ when compared to the harddecision decoding (a 2-level quantizer leading to a binary-input binary-output symmetric channel, denoted by $C_{2,2}[2,2]$ ), when viewed in this context. More specifically, assume that an 8-level quantizer is used in the demodulator output, leading to a $C_{2,8}[8,2]$ channel having the complete bipartite graph $K_{2,8}$ as the associated graph. The $C_{2,2}[2,2]$ channel is associated with the graph $K_{2,2}$ which is embedded on a sphere with two regions, denoted by $S(2)$, thus on a surface of genus zero. In [5] it is shown that the $C_{2,8}[8,2]$ channel may be embedded on the following surfaces: sphere with 8 regions, denoted by $S(8)$; torus with 6 regions (genus 1 ), denoted by $T$ (6); two torus with 4 regions (genus 2 ), denoted by $2 T$ (4); and three torus with 2 regions (genus 3), denoted by $3 T$ (2). Since both channels have binary inputs it follows that the signal space has to be partitioned into two decision regions. As a consequence, the $C_{2,2}[2,2]$ channel is embedded on $S(2)$, and the $C_{2,8}[8,2]$ channel is embedded on $3 T$ (2). The most evident topological difference is the genus of the surface.

A great deal of discrete memoryless channels of practical interest are embedded on compact surfaces with genus $g=2,3$, see [5].

On the other hand, in [6] it is shown that the error probability associated with a signal constellation (a set of points) depends on the curvature, $K$, or equivalently, 
on the genus of a surface (surface where the signal constellation lies), and that the best performance is achieved when considering surfaces with constant negative curvature among the possible values taking on by $K,(K<0, K>0$, and $K=0$ ).

To the best of our knowledge this approach was not considered previously in the context of designing signal sets for digital communication systems. The design of signal constellations is strongly dependent on the existence of regular tessellations in the Euclidean or any other homogeneous space. The homogeneous spaces are important for they have rich algebraic structures and geometric properties so far not fully explored in the context of communication and coding theory. The algebraic structures provide the means for systematic devices implementations whereas the geometric properties are relevant mainly with respect to the efficiency of demodulation and decoding processes.

\section{Hyperbolic tessellations}

One of the important algebraic structures in communication theory is the vector space, that is, a space whose metric is compatible with a norm (Euclidean spaces). It is by use of this structure that it is possible to model and to analyze new communications systems, new modulation schemes, signal processing techniques, signal constellations, and so on. On the other hand, the hyperbolic space, locally $(K<0)$ may be seen as a vector space and since the hyperbolic space is complete, simply connected and of constant negative curvature, the vector space structure holds globally. However, the important difference from the Euclidean space is that there is no norm compatible with the Riemannian metric.

In this section, we consider the hyperbolic plane $\mathbb{H}^{2}$ as a space where we may design signal constellations from regular tessellations. One advantage of these tessellations is that they can be analyzed from its associated groups, [2] and [3].

Definition 2.1. A regular tessellation of the hyperbolic plane $\mathbb{H}^{2}$ is a partition of $\mathbb{H}^{2}$ by non-overlapping regular polygons with the same number of sides which intersect entirely on edges or vertices. A regular tessellation in which q regular $p$-gons meet at each vertex is denoted by $\{p, q\}$.

Since the sum of the internal angles of a hyperbolic triangle is less than $\pi$, 
it follows that there exists a hyperbolic tessellation $\{p, q\}$ if and only if $(p-2)(q-2)>4,[3$, pp. 154-156]. Associated with each tessellation $\{p, q\}$ there exists a group called full symmetry group of $\{p, q\}$, and denoted by $[p, q]$. This group is the isometry group of $\mathbb{H}^{2}$, denoted by $\operatorname{Isom}\left(\mathbb{H}^{2}\right)$, generated by reflections in the hyperbolic lines in which the tessellations $\{p, q\}$ self-reflects, [2], that is $[p, q]$ consists of the isometries of $\mathbb{H}^{2}$ leaving $\{p, q\}$ invariant [3]. From [2], the group $[p, q]$ is generated by the reflections $r_{1}, r_{2}$ and $r_{3}$ on the edges of the hyperbolic triangle with angles $\frac{\pi}{2}, \frac{\pi}{p}, \frac{\pi}{q}$. Consequently, the presentation of the group $[p, q]$ associated with the tessellation $\{p, q\}$ is given by

$$
\left\langle r_{1}, r_{2}, r_{3}: r_{1}^{2}=r_{2}^{2}=r_{3}^{2}=\left(r_{2} r_{1}\right)^{p}=\left(r_{3} r_{2}\right)^{q}=\left(r_{1} r_{3}\right)^{2}=e\right\rangle .
$$

Given a tessellation $\{p, q\}$ with group $[p, q]$, its dual tessellation is $\{q, p\}$ with group $[q, p]$. It is immediate that $[p, q]$ and $[q, p]$ are isomorphic, however the tessellations $\{p, q\}$ and $\{q, p\}$ coincide if and only if $p=q$. The case $p=q$ is called self-dual and if $p=q=4 g$ for an integer $g \geq 2$, then the tessellation $\{4 g, 4 g\}$, by a suitable identification of its edges, yields a universal covering for $\mathbb{H}^{2}$ as an oriented compact surface of genus $g$ and its group [ $\left.4 g, 4 g\right]$ has as a normal subgroup, the fundamental group of this surface, defined by

$$
\pi_{g}=\left\langle a_{1}, \ldots, a_{g}, b_{1}, \ldots, b_{g}: \prod_{i=1}^{g}\left[a_{i}, b_{i}\right]=1\right\rangle .
$$

From this we have that $\pi_{g} \triangleleft[4 g, 4 g]$ and

$$
[4 g, 4 g]=\pi_{g} \ltimes \mathbb{D}_{4 g},
$$

where $\ltimes$ denotes the semidirect product, and $\mathbb{D}_{4 g}$ denotes the dihedral group with $8 g$ elements. The group $[p, q]$ can be obtained as a subgroup of index $2 p$ of the group $\Gamma^{*}(2, p, q)$, called the triangle group which has a triangle $\Delta^{*}$ with angles $\frac{\pi}{2}, \frac{\pi}{p}, \frac{\pi}{q}$ as the fundamental region. Associated with the triangle group is the Fuchsian group, [11] and [12],

$$
\Gamma(2, p, q)=\Gamma^{*}(2, p, q) \cap \operatorname{PSL}(2, \mathbb{R}),
$$

with signature $(0 ; 2, p, q)$ and fundamental region $\Delta^{*} \cup r_{1}\left(\Delta^{*}\right)$. 
Regular tessellations of the form $\{4 g, 4 g\}$ can be obtained as sub-tessellations of the tessellation associated with a triangle group $\Gamma^{*}$ or its Fuchsian part $\Gamma$, with fundamental region $\Delta$ or $\left(\Delta=\Delta^{*} \cup r_{1}\left(\Delta^{*}\right)\right)$ from the determination of the $4 g$-gons formed by the union of copies of $\Delta$ or $\Delta^{*}$ and such that its sides are identified to form an oriented surface of genus $g$. This procedure is equivalent to determining a subgroup of $\Gamma$ isomorphic to $\pi_{g}$, the fundamental group of the compact oriented surface of genus $g$. Hence, the triangle groups are important groups to be employed in the search for relevant tessellations for the purpose of designing signal constellations.

\section{Signal sets matched to groups}

Definition 3.1. A signal set is a discrete subset of the Euclidean or hyperbolic spaces. For each point $s \in S$ the Voronoi region of $s$ is the set $R_{V}(s)=$ $\left\{x \in \mathbb{E}: d(x, s)=\min _{t \in S} d(x, t)\right\}$. A signal set $S$ is matched to a group $G$ if there exists a surjective map $m: G \longrightarrow S$, such that for all $g, g^{\prime} \in G$, $d\left(m(g), m\left(g^{\prime}\right)\right)=d\left(m\left(g^{-1} \cdot g^{\prime}\right), m(e)\right)$. Such an $m$ is called a matched mapping. In addition to that, if $m$ is injective, then $m^{-1}$ is called a matched labelling.

If $m: G \rightarrow S$ is a matched mapping, then $H=m^{-1}(m(e))$ is a subgroup of $G$ and $g \equiv g^{\prime} \bmod H$ if, and only if, $m(g)=m\left(g^{\prime}\right)$. Thus, any matched mapping $m$ corresponds to a bijection $g H \longmapsto m(g)$ of the left cosets of $H$ in $G$ in the elements of $S$. It immediately follows that if $H \triangleleft G$, then the quotient map $m: \frac{G}{H} \rightarrow S$ is a matched labelling. We say that a labelling $m: G \rightarrow S$ is an effective labelling if $H$ does not contain a non-trivial normal subgroup of $G$. In this case, we say that $S$ is effectively matched to $G$. This is the most general situation for if $S$ is not effectively matched to $G$, then considering $H^{\prime}$ as the greatest normal subgroup of $G$ in $H$, results that the map $m: \frac{G}{H^{\prime}} \rightarrow S$ is well-defined, equivalently, $m(g)=m\left(g^{\prime}\right)$ if and only if $g H^{\prime}=g^{\prime} H^{\prime}$ and $g^{\prime} g^{-1} \in H^{\prime} \subseteq H$.

Example 3.1. Let $A$ be a metric space with a left (right) invariant metric $d_{A}$ and $G$ a group with a group metric $d_{G}$ such that there exists a map $m: G \rightarrow A$ which is an isometry. Then for any $g, h \in G$ we have

$$
d_{A}(m(g), m(h))=d_{G}(g, h)=d_{G}\left(g \cdot h^{-1}, e\right)=d_{A}\left(m\left(g \cdot h^{-1}\right), m(e)\right)
$$


resulting that $m$ is a matched labelling.

Theorem 3.1. [8] There exists a matched labelling between a signal set $S$ and a group $G$ if, and only if, $G$ is isomorphic to a transitive subgroup of $\Gamma(S)$, the isometry group of $S$.

In [10] it is shown that certain nonlinear binary codes are the image of linear codes over $\mathbb{Z}_{4}$, allowing in this way more effective decoding procedures. In [9] it is considered the possible extensions of the $\mathbb{Z}_{4}$-linearity to the $G$-linearity, where $G$ is a group.

The next definition of $G$-linear codes encompasses the definition presented in [9], and it fits nicely with the purposes of this paper.

Definition 3.2. A code $C \subseteq S^{I}$ is $G$-linear if there exists an isometry $\mu: G \rightarrow S$, a group code $\mathcal{D} \leq G^{I}$, and a permutation $\sigma \in S^{I}$ such that $\sigma(C)=\mu(\mathcal{D}), \mu$ also denotes the extension $\mu: G^{I} \rightarrow S^{I}$.

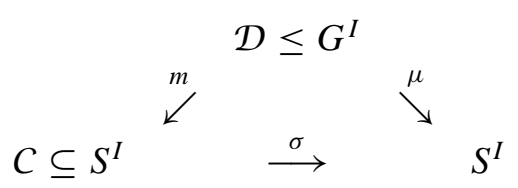

Considering the map $m=\sigma^{-1} \circ \mu: G^{I} \rightarrow S^{I}$ we have:

$$
\begin{aligned}
d_{S^{I}}\left(m(g), m\left(g^{\prime}\right)\right) & =d_{S^{I}}\left(\sigma^{-1}(\mu(g)), \sigma^{-1}\left(\mu\left(g^{\prime}\right)\right)\right) \\
& =d_{S^{I}}\left(\mu(g), \mu\left(g^{\prime}\right)\right)=d_{G^{I}}\left(g, g^{\prime}\right)=d_{G^{I}}\left(g \cdot g^{\prime-1}, e\right) \\
& =d_{S^{I}}\left(\mu\left(g g^{-1}\right), \mu(e)\right)=d_{S^{I}}\left(\sigma^{-1} \mu\left(g g^{\prime-1}\right), \sigma^{-1} \mu(e)\right) \\
& =d_{S^{I}}\left(m\left(g g^{\prime-1}\right), m(e)\right),
\end{aligned}
$$

implying that $m: G^{I} \rightarrow S^{I}$ is a matched labelling, and so is $m: \mathcal{D} \rightarrow C$. From Theorem 3.1, $\mathcal{D}$ is isomorphic to the transitive group of symmetries of $C$, denoted by $\Gamma(C)$.

\section{Geometrically uniform signal sets}

Definition 4.1. [7] A signal set $S$ is called geometrically uniform if the action of the group of symmetries of $S, \Gamma(S)$ on $S$ is transitive. More explicitly, for every 
pair $x, y \in S$, there exists an isometry $u \in \Gamma(S)$ such that $u(x)=y$ leaving $S=\{u(x): u \in \Gamma(S)\}$ invariant. A group $U$ that is minimal for the property that $S=\{u(x): u \in U\}$ is called the generator group of $S$.

Example 4.1. Consider the tessellation $\{8,8\}$ in the hyperbolic plane $\mathbb{H}^{2}$. Then the set $S$ consisting of the center of mass of the octagons of the tessellation (or equivalently, the vertices of the octagons of the dual tessellation) is geometrically uniform, since for each fixed $x \in S$, we have $S=\{T(x): T \in[8,8]\}$. Since $[8,8]=\pi_{8} \ltimes \mathbb{D}_{8}$, where $\mathbb{D}_{8}=\mathbb{Z}_{8} \ltimes \mathbb{Z}_{2}$, it follows that $\mathbb{D}_{8}(x)=\{T(x): x \in$ $\left.\mathbb{D}_{8}\right\}=P$ is an octagon (dual), and $P$ is itself geometrically uniform with $\Gamma(P)=\mathbb{D}_{8}$. Note that $|P|=8$ and $\left|\mathbb{D}_{8}\right|=16$. Thus, $\Gamma(P)$ has more elements than needed to generate $P$. However, considering the subgroups $G_{1}=\mathbb{Z}_{8}$ and $G_{2}=\mathbb{Z}_{4} \ltimes \mathbb{Z}_{2}$ in $\mathbb{D}_{8}$ then, we have $P=G_{1}(x)=G_{2}(x)$. Since $G_{1}$ and $G_{2}$ are not isomorphics and they are proper subgroups of $\mathbb{D}_{8}$, we conclude that either a signal set may have a group of symmetries with more elements than that of the signal set itself, or may have a symmetry group with the same number of elements, however with non-isomorphic groups.

Slepian, [13], has provided an example of a signal set $S$ with 10 signals in $\mathbb{R}^{5}$ that is not the orbit of any subgroup of symmetries of $\mathbb{R}^{5}$. Therefore, not all signal sets, even Euclidean ones, is geometrically uniform.

Definition 4.2. Given a signal set $S$, a subgroup $U(S)$ of $\Gamma(S)$ is a generator group of $S$, if $S=\left\{u\left(s_{0}\right): u \in U(S)\right\}$, for a fixed $s_{0}$ in $S$, and $U(S)$ is minimal for the generation of $S$ in the sense that the map $m: U(S) \rightarrow S, m(u)=u\left(s_{0}\right)$ is a bijection.

Clearly, $m$ induces in $S$ a group structure isomorphic to the one of $U(S)$.

Theorem 4.1. Let $S$ be a signal set, then the following statements are equivalents:

(i) S is geometrically uniform;

(ii) There exists a matched labelling between $S$ and $U(S)$;

(iii) $S$ is $U(S)$-linear with $m: U(S) \rightarrow S$. 
Proof. $\quad(i) \Longleftrightarrow$ (ii) follows from Theorem 3.1, and $(i) \Longleftrightarrow$ (iii) follows immediately from the remark just after Definition 3.2.

Lemma 4.1. Let $S$ be a geometrically uniform signal set, and $x$ and $y$ any two signals in $S$. Then there exists an isometry $u \in \Gamma(S)$ such that $u(x)=y$, and $u\left(R_{V}(x)\right)=R_{V}(y)$. In other words, the Voronoi regions are all congruents.

Proof. $\quad v \in R_{V}(x)$ if, and only if, $d(x, v)=\min _{z \in S}\{d(z, v)\}$ but

$$
\begin{aligned}
d(u(v), y) & =d(u(v), u(x))=d(v, x)=\min _{z \in S}\{d(v, z)\} \\
& =\min _{z \in S}\{d(u(v), u(z))\}=\min _{w \in S}\{d(u(v), w)\} .
\end{aligned}
$$

Hence, $u(v) \in R_{V}(y)$ and $u\left(R_{V}(x)\right) \subseteq R_{V}(y)$. Considering $u^{-1}$, we obtain the inequality in the reverse order, and so $u\left(R_{V}(x)\right)=R_{V}(y)$.

If a hyperbolic signal set is designed from the tessellation $\{p, q\}$, then it is immediate that each polygon is a Voronoi region. In the general case of a Fuchsian group $\Gamma$ (a subgroup of the automorphisms of $\mathbb{H}^{2}$, is the universal covering of $\mathbb{H}^{2} / \Gamma$ ), a Voronoi region is the Dirichlet region, [12].

The congruence of the Voronoi regions is responsible for establishing the relevant properties associated with the signal sets, namely: the global distance profile and the error probability of each signal in the constellation is locally determined.

\section{Hyperbolic geometrically uniform partitions}

The concept of geometrically uniform partitions was introduced by Forney [7], in the context of Euclidean signal sets. As previously mentioned, although the hyperbolic isometry groups have a greater complexity when compared to the Euclidean isometry groups, it is possible to extend to the former the concept of geometrically uniform partitions, as we show in this and in the next sections. For a detailed exposition, we refer the reader to [4].

Definition 5.1. Let $S$ be a geometrically uniform signal set, say $S=\left\{u\left(s_{0}\right)\right.$ : $u \in U(S)\}$, for some fixed $s_{0}$ in $S$. Let $U^{\prime}$ be a normal subgroup of $U(S)$, 
generator group of the signal set $S$. Denoting the orbit of $U^{\prime}$ by $S^{\prime}$, where $S^{\prime}=\left\{u(s): u \in U^{\prime}\right\}$, it follows that if

$$
U=U^{\prime} \cup U^{\prime} a \cup U^{\prime} b \cup \ldots
$$

is a decomposition of $U$ in cosets of $U^{\prime}$, then the partition of $S$ is given by

$$
S=U^{\prime} s_{0} \cup U^{\prime} a s_{0} \cup U^{\prime} b s_{0} \ldots,
$$

denoted by $S / S^{\prime}$, and it is called a geometrically uniform partition. We denote $U\left(S^{\prime}\right)$ by $U^{\prime}$.

Definition 5.2. We call a geometrically uniform partition of a signal set $S$ which is geometrically uniform with a generator group $U(S)$ to any partition $S / S^{\prime}$ induced by a normal subgroup $U^{\prime}$ of $U(S)$.

The concept of geometrically uniform partition is important when we take into account the next fundamental result.

Theorem 5.1. [7] If $S / S^{\prime}$ is a geometrically uniform partition, then the elements of $S / S^{\prime}$ are geometrically uniform, mutually congruent and have $U^{\prime}$ as the common generator group.

Proof. Let $\mathcal{A}$ be denoted by $\mathcal{A}=U(S) / U^{\prime}$. If $a \in \mathcal{A}$, then $a=U^{\prime} u_{a}=u_{a} U^{\prime}$ for some $u_{a} \in U(S)$. Let the corresponding element of the partition $S / S^{\prime}$ be denoted by $S^{\prime}(a)$. Thus,

$$
S^{\prime}(a)=u_{a} U^{\prime}\left(s_{0}\right)=\bigcup_{u \in U^{\prime}} u_{a}\left[u\left(s_{0}\right)\right]=u_{a}\left[\bigcup_{u \in U^{\prime}} u\left(s_{0}\right)\right]=u_{a}\left(S^{\prime}\right) .
$$

Therefore, $S^{\prime}(a) \simeq S^{\prime}$ and for every $a \in \mathcal{A}$, the $S^{\prime}(a)$ are all congruents.

On the other hand,

$$
S^{\prime}(a)=U^{\prime} u_{a}\left(s_{0}\right)=\bigcup_{u \in U^{\prime}} u\left[u_{a}\left(s_{0}\right)\right]
$$

is the orbit of $u_{a}\left(s_{0}\right)$ by $U^{\prime}$. Hence, all $S^{\prime}(a)$ are geometrically uniform with a common generator group $U^{\prime}$.

From Theorem 5.1 it makes sense to use the notation $U\left(S^{\prime}\right)$ for $U^{\prime}$. 
Example 5.1. The Ungerboeck partitions [14] are Euclidean geometrically uniform binary partitions associated with $M$-PSK signal sets with $M=2^{k}$ and subgroups (not necessarily normal) of the form $2^{j}$-PSK determining a partition sequence. The polygons $S$ associated with the hyperbolic tessellations (regular $p$-gons) have $\mathbb{D}_{p}$ as their group of symmetries. Hence, they are constellations generated by $p$-PSK $\left(U(S)=p\right.$-PSK $\left.\leq \mathbb{D}_{p}\right)$.

Theorem 5.1 generalizes in a natural way Ungerboeck's construction, by repeated application in a chain

$$
\ldots U\left(S^{\prime \prime}\right) \triangleleft U\left(S^{\prime}\right) \triangleleft U(S) .
$$

This leads naturally to a geometrically uniform chain partition

$$
S / S^{\prime} / S^{\prime \prime} / \ldots
$$

where in each level the partition sets are congruents and have a common generator group.

Example 5.2. In the Euclidean case, if $\Lambda$ is a lattice and $\Lambda^{\prime}$ is a sublattice of $\Lambda$ of finite index, then any signal set $S=\Lambda+a$ is partitioned in $\left|\Lambda / \Lambda^{\prime}\right|$ geometrically uniform subsets $\Lambda^{\prime} a+v$ with $v \in\left[\Lambda / \Lambda^{\prime}\right]$ for some complete set of representatives $\left[\Lambda / \Lambda^{\prime}\right]$ of $\Lambda$ modulo $\Lambda^{\prime}$.

In the hyperbolic case we have some points to consider: Since the dual tessellation is generated by translations, then an equivalent condition to $T(\Lambda)=\Lambda$, where $T$ denotes translation, is exactly the fact that the tessellation be self-dual. This is equivalent to being of the type $\{p, p\}$. In general, the group generated by translations may have elements of finite order other than the neutral element. In the self-dual case, since the group $\pi_{g}$ has only the relation $\left(\prod\left[a_{i}, b_{i}\right]=1\right)$, it follows that $\pi_{g}$ has only the neutral element with finite order.

\section{Isometric labelling}

Definition 6.1. Let $S / S^{\prime}$ be a geometrically uniform partition. We say that a group $\mathcal{A}$ is a label group for $S / S^{\prime}$ if there exists an isomorphism

$$
m: \mathcal{A} \rightarrow \frac{U(S)}{U\left(S^{\prime}\right)},
$$


$m$ is called a labelling isomorphism. The bijective map

$$
m: \mathcal{A} \rightarrow \frac{S}{S^{\prime}}
$$

defined by the composition of the labelling isomorphism with the bijection

$$
\frac{U(S)}{U\left(S^{\prime}\right)} \rightarrow \frac{S}{S^{\prime}}
$$

is called an isometric labelling of the subsets of $S$ belonging to the partition $S / S^{\prime}$.

The previous definition may be visualized in the next diagram:

$$
\begin{aligned}
& \mathcal{A} \longrightarrow \frac{U(S)}{U\left(S^{\prime}\right)} \longrightarrow \frac{S}{S^{\prime}} \\
& a \longmapsto u_{a} U^{\prime} \longmapsto m(a)=u_{a}\left(S^{\prime}\right)=\left\{u_{a} u\left(s_{0}\right): u \in U^{\prime}\right\} .
\end{aligned}
$$

The fact that $m$ is a well-defined function is a consequence of the fact that if $u_{a} U^{\prime}=v U^{\prime}$ then $u_{a}^{\prime} v^{-1} \in U^{\prime}=U\left(S^{\prime}\right)$. Hence, $u_{a} v^{-1}\left(S^{\prime}\right)=S^{\prime}$. Therefore, $u_{a}\left(S^{\prime}\right)=v\left(S^{\prime}\right)$.

The following properties are immediate:

(i) $m\left(e_{\mathcal{A}}\right)=S^{\prime}$, where $e_{\mathcal{A}}$ denotes the identity element of $\mathcal{A}$;

(ii) $\left|\frac{S}{S^{\prime}}\right|=\left|\frac{U(S)}{U\left(S^{\prime}\right)}\right|=|\mathcal{A}|$.

A partition $S / S^{\prime}$ admits an isometric labelling by a group $\mathcal{A}$ if

(a) $S$ is geometrically uniform;

(b) The subsets of the geometrically uniform partitions are mutually congruents;

(c) There exist isometry groups $U(S)$ and $U\left(S^{\prime}\right)$ such that $U(S)$ generates $S$, $U\left(S^{\prime}\right)$ generates $S^{\prime}, U\left(S^{\prime}\right) \triangleleft U(S)$ and $\mathcal{A} \simeq \frac{U(S)}{U\left(S^{\prime}\right)}$.

Theorem 6.1. A bijective labelling map $m: \mathcal{A} \longrightarrow S / S^{\prime}$ is an isometric labelling if and only if for every $a \in A$ there exists an isometry $u_{a}: \mathcal{A} \longrightarrow \mathcal{A}$ such that for every $b \in \mathcal{A}, m(a b)=u_{a}(m(b))$. 
Proof. If $m: \mathcal{A} \longrightarrow S / S^{\prime}$ is an isometric labelling, from the isomorphism $\mathcal{A} \simeq \frac{U(S)}{U\left(S^{\prime}\right)}$ we have $a b \longmapsto u_{a b} U^{\prime}$ and $a b \longmapsto u_{a} U^{\prime} u_{b} U^{\prime}=u_{a} u_{b} U^{\prime}$ where we obtain

$$
\begin{aligned}
u_{a b} U^{\prime}=u_{a} u_{b} U^{\prime} & =\left\{\left(u_{a} u_{b}\right) u\left(s_{0}\right): u \in U^{\prime}=U\left(S^{\prime}\right)\right\} \\
& =\left\{u_{a}\left[u_{b} u\left(s_{0}\right)\right]: u \in U\left(S^{\prime}\right)\right\}=u_{a}[m(b)] .
\end{aligned}
$$

Conversely, if $m(a b)=u_{a}(m(b))$, for every $a, b \in \mathcal{A}$, then $u_{a b}\left(S^{\prime}\right)=u_{a} u_{b}\left(S^{\prime}\right)$. This implies that $m(a b)=m(a) m(b)$, where $m$ is a homomorphism.

\section{Geometrically uniform signal space codes}

The next definition establishes what we mean by a geometrically uniform signal space code.

Definition 7.1. Let $(\mathcal{A}, *)$ be a group and $I \subseteq \mathbb{Z}$ (eventually finite). Consider the sequence space $\mathcal{A}^{I}=\left\{\left\{a_{k}\right\}_{k \in I}: a_{k} \in \mathcal{A}, \forall k \in I\right\}$, where $\mathcal{A}$ denotes the alphabet and I an index set. Consider the natural group structure in $\mathcal{A}^{I}$. Given a signal set $S$, a geometrically uniform partition $S / S^{\prime}$, and a label set $\mathcal{A}$, we extend in a natural way the isometric labelling map to $\underline{m}: \mathcal{A}^{I} \rightarrow\left(S / S^{\prime}\right)^{I}$. We call a labelling code to any subset $\mathcal{D} \subseteq \mathcal{A}^{I}$. With these notations, the generalized coset code (signal space code) is established as $C\left(S / S^{\prime}, \mathcal{D}\right)=\bigcup_{c \in \mathcal{D}} \underline{m}(c)$.

Since

$$
C\left(S / S^{\prime}, \mathcal{D}\right)=\bigcup_{c \in \mathcal{D}} \underline{m}(c)=\bigcup_{c \in \mathcal{D}}\left\{m\left(c_{k}\right)\right\}_{k \in I},
$$

we have $C\left(S / S^{\prime}, \mathcal{D}\right) \subseteq S^{I}$ is a signal sequence, $s \in S^{I}$ is a code sequence or an element of $C\left(S / S^{\prime}, \mathcal{D}\right)$, if there exists some $c \in \mathcal{D}$ such that $s_{k} \in m\left(c_{k}\right)$ for all $k \in I$. If $S \subseteq \mathbb{R}^{n}$, then $C\left(S / S^{\prime}, \mathcal{D}\right) \subseteq\left(\mathbb{R}^{n}\right)^{I}$. Since $\mathbb{H}$ is not a vector space, there is no definite form for the product.

The next results describe how should an encoder be designed for the hyperbolic signal space codes.

Let $S / S^{\prime}$ be a geometrically uniform partition generated by a partition whose group generators are $U(S)$ and $U\left(S^{\prime}\right)$, and $\mathcal{A} \simeq U(S) / U\left(S^{\prime}\right)$ is the label group. 
Definition 7.2. Let $S / S^{\prime}$ be a geometrically uniform partition with label group $\mathcal{A}$ and $\mathcal{D} \subseteq \mathcal{A}^{I}$ be a normal subgroup $\left(\mathcal{D} \triangleleft \mathcal{A}^{I}\right.$ ), then a generalized coset code is the subset

$$
C\left(S / S^{\prime}, \mathcal{D}\right)=\{\underline{m}(c): c \in \mathcal{D}\} \subseteq S^{I} .
$$

The next theorem provides a connection between $G$-linearity and the generalized coset codes.

Theorem 7.1. Under the hypothesis of Definition 7.2, a generalized coset code is a $U(S)$-linear code.

Proof. Since $S$ is geometrically uniform, let the labelling be given by

$$
\begin{gathered}
\mu: G=U(S) \longrightarrow S \\
u \longmapsto u\left(s_{0}\right)
\end{gathered}
$$

for some fixed $s_{0}$ in $S$. Denoting

$$
\mathcal{A}=\frac{U(S)}{U\left(S^{\prime}\right)}=\left\{u U\left(S^{\prime}\right)\right\}
$$

and the well-defined labelling by

$$
\begin{gathered}
m: \mathcal{A} \longrightarrow \frac{S}{S^{\prime}} \\
u U\left(S^{\prime}\right) \longmapsto u\left(S^{\prime}\right)
\end{gathered}
$$

(in fact, if $u U\left(S^{\prime}\right)=v U\left(S^{\prime}\right)$, then $u^{-1} v \in U\left(S^{\prime}\right)$. Hence, $u^{-1} v\left(S^{\prime}\right)=S^{\prime}$. Therefore, $u\left(S^{\prime}\right)=v\left(S^{\prime}\right)$ ). Let $H=\left\{u \in G: u U\left(S^{\prime}\right) \in \mathcal{D}\right\}$. Thus, if $u, v \in H$, $u U^{\prime}, v U^{\prime} \in \mathcal{D}$ and since $\mathcal{D} \leq \mathcal{A}$, it follows that $u v^{-1} U^{\prime} \in \mathcal{D}$ and $u v^{-1} \in H$. Since $e \in H$, it follows that $H \leq G$. On the other hand, since

$$
\mu(H)=\left\{u\left(s_{0}\right): u \in H\right\}=\bigcup_{u U^{\prime} \in \mathcal{D}} u\left(S^{\prime}\right)=\bigcup m(c)=C\left(S / S^{\prime}, \mathcal{D}\right),
$$

this shows that $C\left(S / S^{\prime}, \mathcal{D}\right)$ is $U(S)$-linear.

In the hyperbolic context, a generalization of the Euclidean definition of geometrically uniform codes, where the label group $\mathcal{A}$ must be Abelian, we impose the condition that code $\mathcal{D}$ be a normal subgroup of the label group $\mathcal{A}$. 
Lemma 7.1. If $C\left(S / S^{\prime}, \mathcal{D}\right)$ is a generalized coset code, then

$$
\left(\frac{S^{I}}{S^{\prime \prime}}\right) \simeq\left(\frac{S}{S^{\prime}}\right)
$$

is a geometrically uniform partition and $\underline{m}: \mathcal{A}^{I} \rightarrow\left(\frac{S}{S^{\prime}}\right)^{I}$ is an isometric labelling for this partition.

\section{Proof.}

(a) $U\left(S^{I}\right)=U(S)^{I}$. In fact, we have $U\left(S^{I}\right) \subseteq U(S)^{I}$. Now, if $H \leq U(S)^{I}$, then $H=\prod_{k \in I} H_{k}$ where $H_{k} \leq U(S)$ for all $k$, and if $U\left(S^{I}\right)=H \supsetneqq U(S)^{I}$ we have $H_{k} \supsetneqq U(S)$ for any $k \in I$, but then $S=H s_{0}$, contrary to the minimality of $U(S)$. Therefore, $U\left(S^{I}\right)=U(S)^{I}$;

(b) $U\left(S^{\prime}\right)^{I} \triangleleft U(S)^{I}$ follows from $U\left(S^{\prime}\right) \triangleleft U(S)$;

(c) $\underline{m}: \mathcal{A}^{I} \rightarrow\left(\frac{S}{S^{\prime}}\right)^{I}$ is an isometry, where $\frac{U(S)^{I}}{U\left(S^{\prime}\right)^{I}}$ yields a geometrically uniform partition $\frac{S^{I}}{S^{\prime I}}$ with label space $\mathcal{A}^{I} \simeq \frac{U(S)^{I}}{U\left(S^{\prime}\right)^{I}}$.

Lemma 7.2. With the previous notations, if $\mathcal{D} \triangleleft \mathcal{A}^{I}$ then with the induced structures, $\left(S^{\prime}\right)^{I} \leq C\left(S / S^{\prime}, \mathcal{D}\right) \leq S^{I}$, we have the isomorphisms:

$$
\frac{S^{I}}{C\left(S / S^{\prime}, \mathcal{D}\right)} \simeq \frac{\mathcal{A}}{\mathcal{D}} ; \frac{C\left(S / S^{\prime}, \mathcal{D}\right)}{\left(S^{\prime}\right)^{I}} \simeq \frac{\mathcal{D}}{e_{\mathcal{A}^{I}}} \simeq \mathcal{D} ; \frac{S^{I}}{\left(S^{\prime}\right)^{I}} \simeq \frac{\mathcal{A}^{I}}{e_{\mathcal{A}^{I}}} \simeq \mathcal{A}^{I},
$$

that is, the chain partitions of the groups $S^{I} / C\left(S / S^{\prime}, \mathcal{D}\right) /\left(S^{\prime}\right)^{I}$ and $\mathcal{A}^{I} / \mathcal{D} / e_{\mathcal{A}}$ are isomorphics.

Proof. We consider the case $|I|=1$. For the general case, it follows by considering the coordinates.

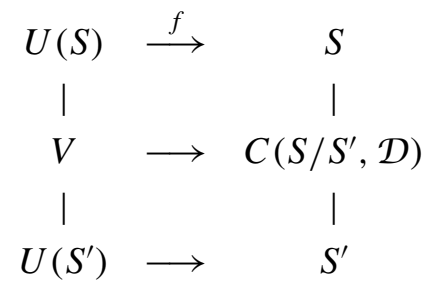


(a) $C\left(S / S^{\prime}, \mathcal{D}\right) \leq S$. If $\phi, \psi \in C\left(S / S^{\prime}, \mathcal{D}\right)$, then there exist $c_{1}, c_{2} \in \mathcal{D}$ such that $\phi \in m\left(c_{1}\right)$ and $\psi \in m\left(c_{2}\right)$. If $f: U(S) \rightarrow S$ is a bijection that induces the group structure in $S$, then there exist $v, w \in U(S)$ such that $f(v)=\phi$ and $f(w)=\psi$. Then $v w \in u_{c_{1}} U^{\prime} \cdot u_{c_{2}} U^{\prime}=u_{c_{1}} u_{c_{2}} U^{\prime}=u_{c_{1} c_{2}} U^{\prime}$, and $f(v w)=\phi \psi \in m\left(c_{1} c_{2}\right)$. Therefore, $\phi \psi \in C\left(S / S^{\prime}, \mathcal{D}\right)$. Since $v U^{\prime}=$ $u_{c_{1}} U^{\prime}$, we have $\left(v U^{\prime}\right)^{-1}=\left(u_{c_{1}} U^{\prime}\right)^{-1}$ or $v^{-1} U^{\prime}=u_{c_{1}}^{-1} U^{\prime}=u_{c_{1}^{-1}} U^{\prime}$. Therefore, $\phi^{-1} \in m\left(c_{1}^{-1}\right) \in C\left(S / S^{\prime}, \mathcal{D}\right)$ and $C\left(S / S^{\prime}, \mathcal{D}\right) \leq S$.

(b) $S^{\prime} \leq C\left(S / S^{\prime}, \mathcal{D}\right)$. Since $C\left(S / S^{\prime}, \mathcal{D}\right)=\bigcup_{c \in \mathcal{D}} m(c)$, we define $V:=$ $f^{-1}\left(C\left(S / S^{\prime}, \mathcal{D}\right)\right)$. This implies, $V \leq U(S)$ and

$$
\begin{aligned}
V & =f^{-1}\left(C\left(S / S^{\prime}, \mathcal{D}\right)\right) \\
& =f^{-1}\left(\bigcup_{c \in \mathcal{D}} m(c)\right)=\bigcup_{c \in \mathcal{D}} f^{-1}(m(c))=\bigcup_{c \in \mathcal{D}} u_{c} U^{\prime} .
\end{aligned}
$$

In particular, $m(1)=U\left(S^{\prime}\right)=U^{\prime}=1 \cdot U^{\prime} \subseteq V$, and it follows that $S^{\prime} \subseteq C\left(S / S^{\prime}, \mathcal{D}\right)$ or $S^{\prime} \leq C\left(S / S^{\prime}, \mathcal{D}\right)$.

(c) $\frac{\mathcal{A}}{\mathcal{D}} \simeq \frac{S}{C\left(S / S^{\prime}, \mathcal{D}\right)}$. Since $V=f^{-1}\left(C\left(S / S^{\prime}, \mathcal{D}\right)\right)$, we have

$$
\frac{U(S)}{V} \simeq \frac{S}{C\left(S / S^{\prime}, \mathcal{D}\right)}
$$

Considering

$$
\Phi: \mathcal{A} \longrightarrow \frac{U(S)}{V}, a \longmapsto u_{a} V
$$

( $\Phi$ is well-defined for $u_{1} U^{\prime}=u_{2} U^{\prime}$ implies $u_{1} u_{2}^{-1} \in U^{\prime} \subseteq V$. Therefore, $u_{1} V=u_{2} V$.), we have $K \operatorname{er} \Phi=\mathcal{D}\left(u_{a} \in V\right.$ if, and only if, $f\left(u_{a}\right) \in m(c)$ for some $c \in \mathcal{D}$ if, and only if, $a \in \mathcal{D}$ ). Therefore,

$$
\begin{aligned}
& \frac{\mathcal{A}}{\mathcal{D}} \simeq \frac{U(S)}{V} \simeq \frac{S}{C\left(S / S^{\prime}, \mathcal{D}\right)} \\
& \mathcal{A} \stackrel{g}{\longrightarrow} \frac{U(S)}{U\left(S^{\prime}\right)} \stackrel{\bar{f}}{\longrightarrow} \frac{S}{S^{\prime}}
\end{aligned}
$$

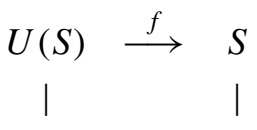


(d) $\mathcal{D} \simeq \frac{\mathcal{D}}{\{e\}} \simeq \frac{C\left(S / S^{\prime}, \mathcal{D}\right)}{S^{\prime}}$. Considering,

$$
g: \mathcal{A} \longrightarrow \frac{U(S)}{U\left(S^{\prime}\right)}
$$

and the label isomorphism as $g(c)=u_{c} U^{\prime}$, we have

$$
g(\mathcal{D})=\frac{V}{U\left(S^{\prime}\right)}
$$

(in fact, if $u_{c} U^{\prime} \in g(\mathcal{D})$, by definition $u_{c} U^{\prime} \subseteq V$, and so $u_{c} U^{\prime} \in \frac{V}{U\left(S^{\prime}\right)}$ then $u U^{\prime}=w U^{\prime}$ and so

$$
w \in \bigcup_{c \in \mathcal{D}} u_{c} U^{\prime} \text { or } w=u_{c} u_{1} \text { for } c \in \mathcal{D} \text { and } u_{1} \in U^{\prime} .
$$

Thus, $\left.g(\mathcal{D})=\frac{V}{U\left(S^{\prime}\right)}\right)$ and

$$
\mathcal{D} \simeq \frac{V}{U\left(S^{\prime}\right)}
$$

Hence, we have $\frac{V}{U\left(S^{\prime}\right)}$ is the image of $C$ by the labelling isomorphism, or equivalently,

$$
V=\bigcup_{u \in g(\mathcal{D})} u U^{\prime}=\bigcup g(\mathcal{D}) .
$$

Letting $f \mid V$ and taking quotients, we have

$$
\frac{V}{U\left(S^{\prime}\right)} \simeq \frac{C\left(S / S^{\prime}, \mathcal{D}\right)}{S^{\prime}},
$$

from (2) and (3) we have then that: $\mathcal{D} \simeq \frac{C\left(S / S^{\prime}, \mathcal{D}\right)}{S^{\prime}}$.

The right cosets of $\mathcal{D}$ in $\mathcal{A}$ (recall that $\mathcal{D} \triangleleft \mathcal{A}$ ) are written in the form $\mathcal{D} \cdot a$, where $a \in \mathcal{A}$ is arbitrary. For such a class $\mathcal{D} \cdot a$, the labelling $m: \mathcal{A} \longrightarrow \frac{S}{S^{\prime}}$ defines a subset $C\left(S / S^{\prime}, \mathcal{D} \cdot a\right)$ of $S^{I}$ called a label translate of $C\left(S / S^{\prime}, \mathcal{D}\right)$, that is,

$$
C\left(S / S^{\prime}, \mathcal{D} \cdot a\right)=\bigcup_{c \in \mathcal{D}} \underline{m}(c \cdot a) .
$$


Lemma 7.3. The label translates of $C\left(S / S^{\prime}, \mathcal{D}\right)$ are the right cosets of $C\left(S / S^{\prime}\right.$, $\mathcal{D})$ in $S^{I}$ under the induced group structure.

Proof. We consider the case $|I|=1$. For the general case, it follows by considering the coordinates. Let

$$
\begin{aligned}
f^{-1}(C & \left.\left(S / S^{\prime}, \mathcal{D} \cdot a\right)\right)=f^{-1}\left(\bigcup_{c \in \mathcal{D}} \underline{m}(c \cdot a)\right)=\bigcup_{c \in \mathcal{D}} f^{-1}(\underline{m}(c \cdot a))=\bigcup_{c e \mathcal{D}} u_{c \cdot a} U^{\prime} \\
= & \bigcup_{c \in \mathcal{D}} u_{c} u_{a} U^{\prime}=\left[\bigcup_{c \in \mathcal{D}} u_{c} U^{\prime}\right] u_{a}=\left[\bigcup_{c \in \mathcal{D}} f^{-1}(\underline{m}(c))\right] f^{-1}\left(f\left(u_{a}\right)\right) \\
= & {\left[f^{-1}\left(\bigcup_{c \in \mathcal{D}}(\underline{m}(c))\right] f^{-1}\left(f\left(u_{a}\right)\right)=f^{-1}\left[\left[\bigcup_{c \in \mathcal{D}} \underline{m}(c)\right] f\left(u_{a}\right)\right]\right.} \\
= & f^{-1}\left[\left[C\left(S / S^{\prime}, \mathcal{D} \cdot a\right)\right] f\left(u_{a}\right)\right] .
\end{aligned}
$$

It follows that $C\left(S / S^{\prime}, \mathcal{D} \cdot a\right)=C\left(S / S^{\prime}, \mathcal{D}\right) \cdot f\left(u_{a}\right)$.

The previous results assure us the validity of the following version of Forney's Theorem on the generalized coset codes.

Theorem 7.2. If $C\left(S / S^{\prime}, \mathcal{D}\right)$ is a generalized coset code, then $S^{I} / C\left(S / S^{\prime}, \mathcal{D}\right) /$ $\left(S^{\prime}\right)^{I}$ is a geometrically uniform partition chain and the label translate $C\left(S / S^{\prime}\right.$, $\mathcal{D} \cdot a)$ of $C\left(S / S^{\prime}, \mathcal{D}\right)$ are geometrically uniform, mutually congruent and have common symmetry group $U\left(C\left(S / S^{\prime}, \mathcal{D}\right)\right)=V$.

Corollary 7.1. If $C\left(S / S^{\prime}, \mathcal{D}\right)$ is a (translate of a) generalized coset code, then: (a) The Voronoi regions associated with two code sequences $s, s^{\prime} \in C\left(S / S^{\prime}, \mathcal{D}\right)$ are congruents; (b) The distance profile $D P(s)=\left\{\left\|s-s^{\prime}\right\|: s^{\prime} \in C\left(S / S^{\prime}, \mathcal{D}\right)\right\}$ from a given signal point $s \in C\left(S / S^{\prime}, \mathcal{D}\right)$ to the remaining points $s^{\prime} \in C$ $\left(S / S^{\prime}, \mathcal{D}\right)$ is independent of $s$.

Example 7.1. With the notations introduced in Theorem 7.1, we define, for an arbitrary $s_{0}$ in $\mathbb{H}^{2}$, the signal set $S=[15,3]\left(s_{0}\right)$ and the subset $S^{\prime}=P\left(s_{0}\right)$. As a 
consequence, we have $U(S)=[15,3]$, and $U\left(S^{\prime}\right)=P$. From Theorem 7.1, the label group

$$
\mathcal{A}=\frac{U(S)}{U\left(S^{\prime}\right)}=\left\{P ; r_{1} P\right\} \simeq \mathbb{Z}_{2},
$$

where we denote $\mathbb{Z}_{2}=\left\{1, r_{1}\right\}$. With the notations introduced in Definitions 6.1 and 7.2, the labelling

$$
m: \mathcal{A} \longrightarrow \frac{S}{S^{\prime}}
$$

have the form $m(1)=s_{0} \bmod P$, and $m\left(r_{1}\right)=r_{1}\left(s_{0}\right) \bmod P$. Now, taking $I=\{1,2\}$, we have the natural extension for the labelling

$$
m: A^{2} \longrightarrow\left(\frac{S}{S^{\prime}}\right)^{2}
$$

Denoting $A^{2}=\left\{(1,1) ;\left(1, r_{1}\right) ;\left(r_{1}, 1\right) ;\left(r_{1}, r_{1}\right)\right\}$, and considering the label code $\mathcal{D}_{1}=\left\{(1,1) ;\left(1, r_{1}\right)\right\}$, we have the signal space code:

$$
C\left(S / S^{\prime}, \mathcal{D}_{1}\right)=\bigcup_{c \in \mathcal{D}}\{m(c)\},
$$

so $s=\left(s_{1}, s_{2}\right) \in C\left(S / S^{\prime}, \mathcal{D}_{1}\right)$ if, and only if, there exists $\left(c_{1} ; c_{2}\right) \in \mathcal{D}_{1}$ such that $s_{i} \in m\left(c_{i}\right)$ for $i=1,2$. For the case in consideration, $c_{1}=1$, and it follows that $s \in S^{\prime} \times S^{\prime}$ or $s \in S^{\prime} \times r_{1}\left(S^{\prime}\right)$. Therefore, $C\left(S / S^{\prime}, \mathcal{D}_{1}\right)=S^{\prime} \times S^{\prime} \cup S^{\prime} \times r_{1}\left(S^{\prime}\right)$. In the same way, defining $\mathcal{D}_{2}=\left\{(1,1) ;\left(r_{1}, 1\right)\right\}$ and $\mathcal{D}_{3}=\left\{(1,1) ;\left(r_{1}, r_{1}\right)\right\}$, we obtain the signal space codes $C\left(S / S^{\prime}, \mathcal{D}_{2}\right)=S^{\prime} \times S^{\prime} \cup r_{1}\left(S^{\prime}\right) \times S^{\prime}$ and $C\left(S / S^{\prime}, \mathcal{D}_{3}\right)$ $=S^{\prime} \times S^{\prime} \cup r_{1}\left(S^{\prime}\right) \times r_{1}\left(S^{\prime}\right)$, respectively.

Example 7.2. Considering the group

$$
\begin{aligned}
& A^{3}=\left\{(1,1,1) ;\left(1,1, r_{1}\right) ;\left(1, r_{1}, 1\right) ;\left(1, r_{1}, r_{1}\right) ;\right. \\
&\left.\left(r_{1}, 1,1\right) ;\left(r_{1}, 1, r_{1}\right) ;\left(r_{1}, r_{1}, 1\right) ;\left(r_{1}, r_{1}, r_{1}\right)\right\},
\end{aligned}
$$

and the label group

$$
\mathcal{D}_{1}=\left\{(1,1,1) ;\left(1,1, r_{1}\right) ;\left(1, r_{1}, 1\right) ;\left(1, r_{1}, r_{1}\right)\right\},
$$

similarly to the previous procedure, the following signal space code is obtained $C\left(S / S^{\prime}, \mathcal{D}_{1}\right)=S^{\prime} \times S^{\prime} \times S^{\prime} \cup S^{\prime} \times S^{\prime} \times r_{1}\left(S^{\prime}\right) \cup S^{\prime} \times r_{1}\left(S^{\prime}\right) \times S^{\prime} \cup S^{\prime} \times r_{1}\left(S^{\prime}\right) \times r_{1}\left(S^{\prime}\right)$. 


\section{Conclusions}

Since there are discrete memoryless communication channels of practical interest which may be embedded on surfaces with genus $g=0,1,2$, and 3 , and in addition to this, the design of geometrically uniform signal sets and codes were extensively considered only for the cases $g=0$ and 1 . Hence, comes the question: What should be the conditions to be followed in order to design geometrically uniform signal sets and codes when $g=2$ and 3 ? The answer to this question was the aim of this paper where we considered both the concepts of regular tessellations $\{p, q\}$ and the corresponding full symmetry group $[p, q]$ and its subgroups in the hyperbolic plane which are necessary to the establishment of the extension of the concept of geometrically uniform signal sets, partitions and signal space codes to the hyperbolic plane.

\section{REFERENCES}

[1] E. Biglieri, Performance Evaluation of Digital Communication Schemes Based on Generalized Concatenation, Politecnico di Torino, Torino, (1991).

[2] H.M.S. Coxeter and W.O.J. Moser, Generators and Relations for Discrete Groups, Berlin, Springer, (1965).

[3] P.A. Firby and C.F. Gardiner, Surface Topology, New York, Ellis Horwood, (1991).

[4] H. Lazari, A Contribution to the Hyperbolic Geometrically Uniform Codes, Ph.D. Dissertation, DT-FEEC-UNICAMP, Feb. 2000 (in portuguese).

[5] J.D. Lima and R. Palazzo Jr., "Embedding discrete memoryless channels on compact and minimal surfaces”, IEEE Information Theory Workshop, India 2002.

[6] R.G. Cavalcante, Performance Analysis of Signal Constellations in Riemannian Varieties, MS Thesis, FEEC-UNICAMP, Brazil, (2002) (in portuguese).

[7] G.D. Forney Jr., "Geometrically uniform codes", IEEE Trans. Inform. Theory, vol. IT 37 no. 5, pp. 1241-1260, Sep. (1991).

[8] H.A. Loeliger, "Signal sets matched to groups", IEEE Trans. Inform. Theory, vol. IT-37, no. 6, pp. 1675-1682, Nov. (1991).

[9] J.R. Gerônimo, R. Palazzo Jr., S.I.R. Costa, J.C. Interlando and P. Brumatti, “On the existence of $\mathbb{Z}_{4} \times \mathbb{Z}_{2}^{k-2}$-linear binary codes from the nonexistence of $\mathbb{Z}_{2^{k}}$-linear binary codes", Preprint.

[10] A.R. Hammons Jr., V. Kumar, A.R. Calderbank, N.A.J. Sloane and P. Solé, “The $\mathbb{Z}_{4}$-linearity of Kerdock, Preparata, Goethals and related codes", IEEE Trans. Inform. Theory, 40 no. 2, p. 301-319, March (1994). 
[11] S. Katok, Fuchsian Groups, Chicago, University of Chicago Press, (1992).

[12] W. Magnus, A. Karras and D. Solitar, Combinatorial Group Theory, New York, Interscience, (1966).

[13] D. Slepian, "Group codes for the gaussian channel”, Bell Sys. Tech. Journal, v. 37 (1968), p. $575-602$.

[14] G. Ungerboeck, "Channel coding with multilevel/phase signals", IEEE Trans. Inform. Theory, vol. IT 28 no.1, pp.55-67, Jan. (1982). 